\title{
Diffusing-wave polarimetry for tissue diagnostics
}

Callum Macdonald, Alexander Doronin, Adrian F. Peña, Michael Eccles, Igor Meglinski

Callum Macdonald, Alexander Doronin, Adrian F. Peña, Michael Eccles, Igor Meglinski, "Diffusing-wave polarimetry for tissue diagnostics," Proc. SPIE 8940, Optical Biopsy XII, 894007 (17 March 2014); doi: 10.1117/12.2038658

SPIE. Event: SPIE BiOS, 2014, San Francisco, California, United States 


\title{
Diffusing-Wave Polarimetry for tissue diagnostics
}

\author{
Callum Macdonald ${ }^{a}$, Alexander Doronin $^{a}$, Adrian F. Peña $^{a}$, Michael Eccles $^{b}$ and Igor Meglinski ${ }^{a}$

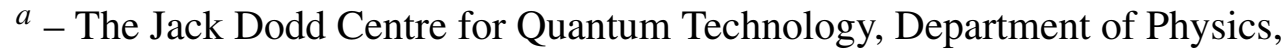 \\ University of Otago, Dunedin, New Zealand \\ $b$ - Department of Pathology, Dunedin School of Medicine, \\ University of Otago, Dunedin, New Zealand
}

\begin{abstract}
We exploit the directional awareness of circularly and/or elliptically polarized light propagating within media which exhibit high numbers of scattering events. By tracking the Stokes vector of the detected light on the Poincare sphere, we demonstrate its applicability for characterization of anisotropy of scattering. A phenomenological model is shown to have an excellent agreement with the experimental data and with the results obtained by the polarization tracking Monte Carlo model, developed in house. By analogy to diffusing-wave spectroscopy we call this approach diffusing-wave polarimetry, and illustrate its utility in probing cancerous and non-cancerous tissue samplesin vitro for diagnostic purposes.
\end{abstract}

Keywords: Circular polarization, Poincaré sphere, Stokes vector, Monte Carlo, helicity, optical biopsy, cancer diagnostics, polarimetry

\section{INTRODUCTION}

Optical techniques have long been of interest for a number of diagnostic applications including biomedicine, as they have a unique ability to observe a wide range of structural, dynamic and functional properties of the medium being probed. This analysis can be carried out at micro- and macroscopic levels in situ, non-invasively, and in real-time, adding to it's attractiveness. Recently, optical modalities utilizing the polarization of light have gained much attention, especially in the field of biomedical optics. ${ }^{1}$ A number of studies have already taken advantage of polarization information contained within scattered light, with the techniques such as polarization discrimination, ellipsometry, and Muller matrix imaging. ${ }^{1-3}$ Traditional polarimetry and ellipsometry methods are used for characterization of transparent media and thin films, while Mueller Matrix-based polarimetry is applied to image thin tissue samples and characterize the depolarizing effect a sample has on incident light. ${ }^{2,3}$ The depolarization rate depends strongly on the size and shape of scattering particles, ${ }^{4,5}$ as well as on the number of scattering events. ${ }^{6}$

A popular belief is that in turbid scattering media, linear polarization is better preserved than circular polarization. However, this turns out to be true only when the particle size $d$ is smaller than the wavelength $\lambda$ of incident light. Small light scattering particles $(d<\lambda)$ are better at preserving linear polarization, whereas in media consisting of larger particle sizes $(d \geq \lambda)$, circular polarization is preserved longer. This phenomenon, known as polarization memory, ${ }^{7-9}$ was explained in terms of specific features of the Mie tensor phase function, ${ }^{10}$ and allows for the scattering length required to randomize the helicity of incident light $l_{x}$ to be greater than the transport mean free path $l_{t r}$.

In the current letter we consider the 'directional awareness' of scattered circularly polarized light and introduce its use for diagnostic purposes. It is well known that when reflected from the medium surface, incident circularly or elliptically polarized light of a given handedness of polarization undergoes a flip in its helicity. ${ }^{11}$ The same goes for backscattered light (Figure 1). Thus, if the angle between the incident $k$ and scattered $k^{\prime}$ wave vectors $\theta=\widehat{k k}^{\prime}>\pi / 2$ the scattered light will have a reversed helicity (Figure 1). While, with $\theta<\pi / 2$ the helicity remains the same. This helicity flip phenomenon is of fundamental importance. Linear polarization possesses no such sense of the direction in which it travels.

Correspondence: Department of Physics, University of Otago, P.O. Box 56, Dunedin, 9054, New Zealand

E-mail: igor.meglinski@otago.ac.nz URL: http://www.biophotonics.ac.nz

Optical Biopsy XII, edited by Robert R. Alfano, Stavros G. Demos, Proc. of SPIE Vol. 8940,

894007 · C 2014 SPIE · CCC code: 1605-7422/14/\$18 · doi: 10.1117/12.2038658 


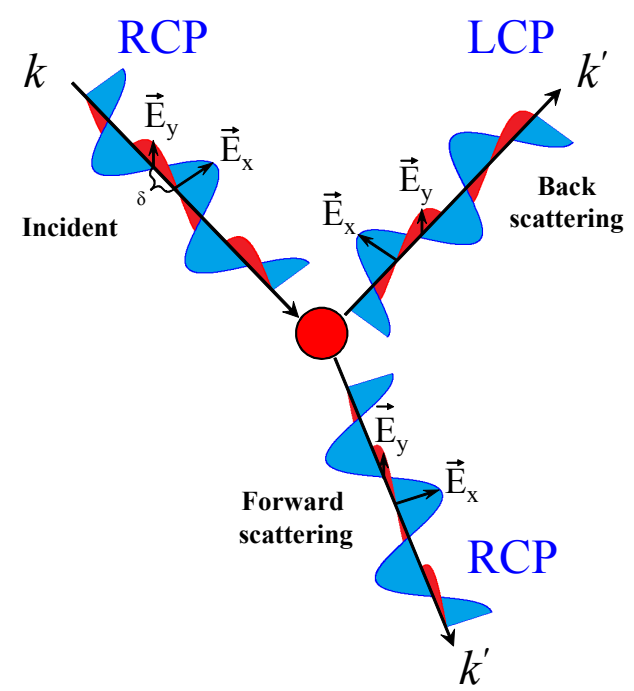

Figure 1. Schematic presentation of forward and backscattered polarized light. When light with right handed circular polarization (RCP) is scattered in the forward direction its helicity is preserved, whereas for backscattered light, the phase shift $\delta$ between the $E_{x}$ and $E_{y}$ field components is reversed, causing the light to switch from right to the left handed circular polarization (LCP).

\section{MATERIALS AND METHODS}

Considering the propagation of circularly or elliptically polarized light through a turbid medium where multiple scattering events occur, light that has backscattered an odd number of times will correspond to a reversal in helicity, and, thus, contribute the cross polarized portion of the detected signal. Whereas, light which experienced an even number of backscattering events contributes to the co-polarized signal, i.e. an even number of helicity changes have not changed the handedness of the incident light. Assuming that for a photon packet to be detected with a reversed helicity, there are two conditions it requires to be satisfy: firstly, it must undergo an odd number of backscattering events each with $\pi / 2<\theta<3 \pi / 2$ (see Figure 1). Secondly, these scattering events must take the photon packet through the medium in such a way as to allow it to be detected; i.e. exit the medium within the detection zone and with an appropriate trajectory. By multiplying the probability of satisfying the first condition after $N$ scattering events $p_{-}(N)$, with the conditional probability of satisfying the second condition after $N$ scattering events $p_{d}(N) \mid p_{-}(N)$, we get the probability of detecting a photon packet with a reversed helicity as a function of its scattering order $N$. This is represented by $R_{-}(N)$, where

$$
R_{-}(N)=p_{-}(N)\left[p_{d}(N) \mid p_{-}(N)\right] .
$$

Note, the conditional probability must be taken as the two distributions are not independent (the outcome of one event will effect the probability of the other). It follows that the probability of detecting photons with a co-polarized helicity relative to the source is defined as:

$$
R_{+}(N)=p_{+}(N)\left[p_{d}(N) \mid p_{+}(N)\right] .
$$

Assuming that the medium consists of identical non-interacting particles whose anisotropy of scattering can described by the Henyey-Greenstein phase function: ${ }^{12}$

$$
p(\theta)=\frac{1}{4 \pi} \frac{1-g^{2}}{\left(1+g^{2}-2 g \cos \theta\right)^{3 / 2}},
$$

the probability of a photon being backscattered $\left(S_{-}\right)$or forward-scattered $\left(S_{+}\right)$by one of these particles can be found by integrating the phase function over all rear angles, or over all frontal angles. We denote these probabilities, respectively, as:

$$
S_{-}=\int_{\frac{\pi}{2}}^{\frac{3}{2} \pi} p(\theta) d \theta, \quad S_{+}=\int_{-\frac{\pi}{2}}^{\frac{\pi}{2}} p(\theta) d \theta .
$$


Now, the probability of a photon packet being in a reversed helicity state after $N$ scattering events can be found by adding the probabilities of all possible ways the photon packet can be detected in a reversed state:

$$
p_{-}(N)=\sum_{j=1,3,5, \ldots}^{m} \frac{N !}{j !(N-j) !} S_{-}^{j} S_{+}^{N-j},
$$

where the sum is over all odd integers between 1 and $N$, and $m$ is the highest odd integer contained in this set. This function shows the number of scattering events required to randomize a photon's helicity by how long it takes $p_{-}$and $p_{+}$ to be equalized, i.e. to reach a value of 0.5 (note $p_{+}=1-p_{-}$). We refer to the number of scattering events required to achieve this random helicity as $N_{x}$.

Next, we consider the conditional probabilities of detection $p_{d}(N) \mid p_{-}(N)$ and $p_{d}(N) \mid p_{+}(N)$. These distributions are heavily influenced by the geometry of the source and detector as well as by the medium properties. Assuming a photon has randomized it's direction after $N^{\prime}$ scattering events, (i.e. after traveling one transport mean free path) it has an equal probability of being anywhere in the medium at a distance $l_{t r}$ from the point of incidence, regardless of whether it is in a reversed state or not. So, it follows that for all $N$ greater than $N^{\prime}$ the conditional probabilities simply become the probability of detection $p_{d}(N)$ as the two distributions are no longer dependent. Eqs. 1 and 2 then become

$$
R_{-}=p_{-}(N) p_{d}(N), R_{+}=p_{+}(N) p_{d}(N) \text { for } N>N^{\prime} .
$$

For $N<N^{\prime}$, the conditional probabilities must be used, as the probability of detecting a photon at a low scattering order will be largely determined by its helicity. Forward scattered photons will generally overshoot the detector and have much larger pathlengths than reversed photons, which have turned sharply towards the detector generally after just a single backscattering event. Note however that for a source detector separation greater than $l_{t r}$, Eq. 6 is generally valid for all $N$ because no photons are detected with a scattering order less than $N^{\prime}$.

To form the detection probability distribution for a particular experimental setup we use Monte Carlo (MC) modeling tool developed in house. ${ }^{13,14}$ By tracking the random walk of every photon packet the simulation can count the probability of a photon of scattering order $N$ being detected and, thus, assemble a normalized distribution for $p_{d}(N)$.

Integrating $R_{-}$and $R_{+}$over all $N$ we get the probability of detecting a reversed $\left(I_{-}\right)$or non-reversed $\left(I_{+}\right)$portion of light:

$$
I_{-}=\int_{0}^{N_{\max }} R_{-}(N) d N, \quad I_{+}=\int_{0}^{N_{\max }} R_{+}(N) d N,
$$

where $N_{\max }$ is determined solely by absorption in the case of a semi-infinite medium.

Physical polarimetry experiments discussed in the next section were carried out using the experimental apparatus shown in Figure 2. Linear polarized light generated by low coherence $(\approx 100 \mu \mathrm{m})$ laser diode $(30 \mathrm{~mW}, 639 \mathrm{~nm}$, Newport Inc., model LQC639-30C) is directed towards the sample and at $55^{\circ}$ from the normal and circularly RHP light produced by a quarter wave plate is focused on the medium surface. Scattered light is collected by a second lens focused at a distance $d=3.5 \mathrm{~mm}$ away from the point of incidence $\left(d>l_{t r}\right)$. The lens which is angled at $10^{\circ}$ from the normal then passes light into the polarimeter (Thorlabs inc. PAX5710VIS) to be analyzed.

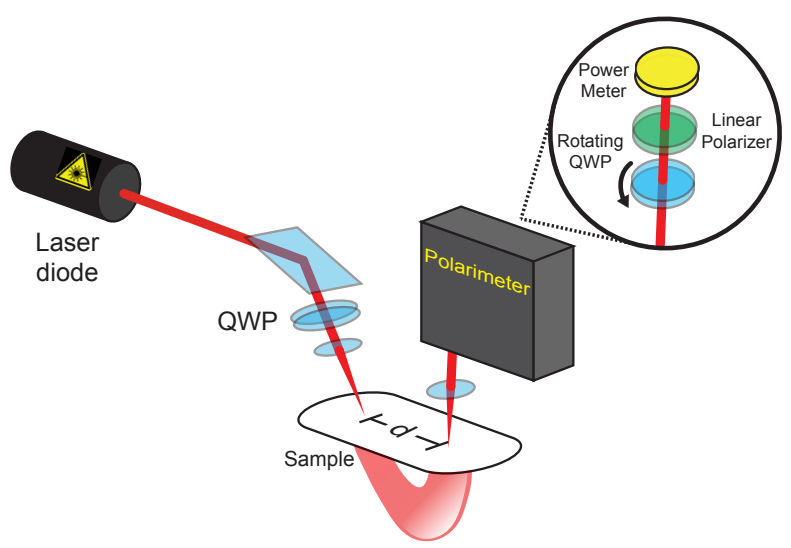

Figure 2. Schematic presentation of the experimental system. 


\section{RESULTS AND DISCUSSION}

Figure 3 shows the relative probabilities

$$
\frac{I_{-}}{I_{-}+I_{+}} \quad, \quad \frac{I_{+}}{I_{-}+I_{+}}
$$

for a range of anisotropy of scattering $-g$ values (red line and blue line respectively). The comparison of the results obtained by this model with the results of a more complex polarization tracking MC simulation ${ }^{15-17}$ is also presented in Figure 3 (respectively, as red squares and blue circles). Here, we implemented MC modeling to track the polarization of each photon as it propagated through the medium. The calculations have been made for a particular experimental geometry shown in Figure 2 and discussed below.

As one can see, the relative probability of detecting a reversed photon decreases with increasing anisotropy $g$ in the range $(0.8<g \leq 1)$; $g$ is the mean cosine of scattering angle, $g=\langle\cos \theta\rangle$. This is as expected as a higher anisotropy causes photons to almost exclusively scatter in a series of forward angles, gradually turning towards the detector. For low anisotropy $(0 \leq g \leq 0.8)$ the relative probabilities are equivalent, this is a result of using the same detection probability distribution in both cases which was the assumption made resulting in Eq.6. The agreement with the polarization tracking MC model shows that this was a valid assumption for cases where the source-detector separation is greater than $l_{t r}$. The outcome of these two modeling approaches can overall be seen to obey the same trend, with the phenomenological model predicting a slightly higher prevalence of light with the same helicity as the source for the media with the scattering particles of high anisotropy.

In order to relate the ratio of RCP and LCP photons to an observable quantity, we consider the superposition of each photon packet and the resulting bulk signal. The final state of polarization for each photon packet can be characterized by a Stokes 4-vector: ${ }^{11}$

$$
\mathbf{S}=\left[\begin{array}{c}
S_{0} \\
S_{1} \\
S_{2} \\
S_{3}
\end{array}\right]
$$

The last element, $S_{3}$, of this vector characterizes it's ellipticity $\chi$, and it's helicity (sign of $\chi$ ):

$$
S_{3}=S_{0} \sin \chi
$$

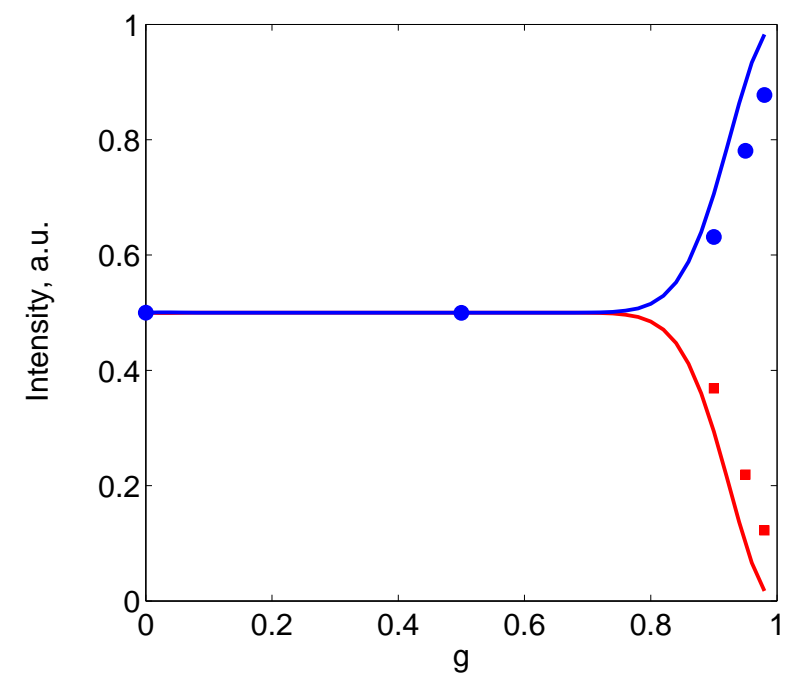

Figure 3. The results of MC modeling showing the relative number of reversed photons (red line), and non-reversed photons (blue line) detected as a function of anisotropy of scattering $g$. Simulated relative number of reversed photons (circles) and non-reversed photons (squares) exiting the medium as a function of the anisotropy $g$. 
With a normalized Stokes vector this quantity has a range $-1 \leq S_{3} \leq 1$, with left handed states of polarisation taking negative values, and right handed states of polarisation taking positive values. The final Stokes vector of the resultant signal is found by the superposition of all photons and can be represented by: ${ }^{18}$

$$
\mathbf{S}=\sum \mathbf{S}_{j}+\sum_{j \neq k} \mathbf{S}_{j k}
$$

where the $\mathbf{S}_{j k}$ are the interference terms for all pairs of photons. This term is explained in depth in, ${ }^{18}$ where it is shown that the magnitude of the interference term is determined by the degree of coherence of the sources and their relative phases, as well as by their relative states of polarization. For completely incoherent sources however this term disappears, and we are left with the simple addition of each individual Stokes vector. For a similar reason if the number of coherent sources of largely varying pathlength differences increases to a very high number, the $\mathbf{S}_{j k}$ term also becomes negligible. Thus, for low coherent light propagating in a highly scattering medium $S_{3}$ is proportional to the ratio of RCP and LCP photons being detected assuming the magnitudes of each $S_{3}$ are equivalently distributed for both negative and positive values. To confirm this we conducted a physical experiment measuring scattering of circularly RCP light in solutions of polystyrene micro-spheres.

Water solutions of polystyrene microspheres (Polysciences, Inc.) of $0.35,0.75$ and $1.5 \mu m$ in diameters have been used in the experiments using the apparatus shown in Figure 2. The concentrations of the polystyrene microspheres in all three solutions have been chosen to match the same scattering coefficient $\mu_{s}=4.57 \mathrm{~mm}^{-1}$. The resulting states of polarization are shown on Figure 4 in comparison with the results of MC simulation. The Poincaré Sphere has been used as a simple graphical tool to observe changes of the Stokes vector for semi-infinite scattering medium. ${ }^{19}$ As one can see the bulk polarization state measured from the medium with the highest scattering anisotropy (1.5 $\mu \mathrm{m}$ spheres) has the highest overall ellipticity, or $S_{3}$ component. The overall trend between ellipticity and changes of anisotropy of scattering is clearly seen between all three samples. These results are well agreed with the the results of MC simulation (see Figure 4) showing the increase of the probability of successive forward scattering events, also predicted by phenomenological model presented above.

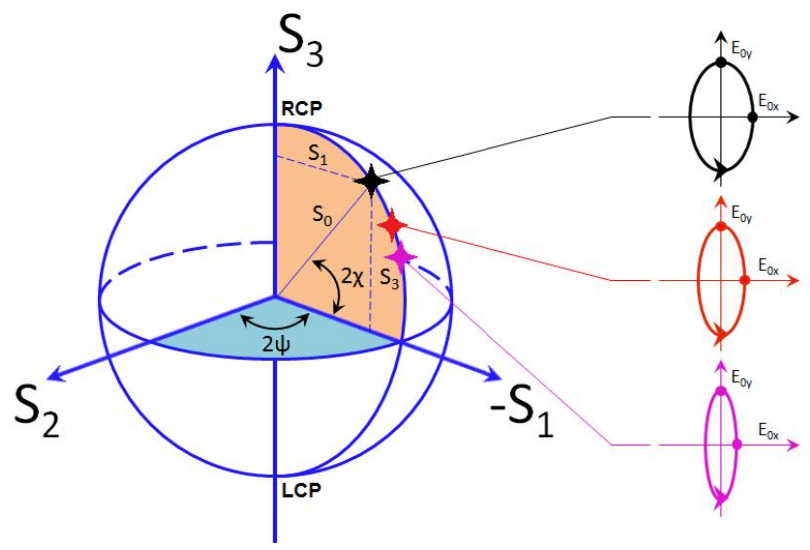

Figure 4. The signature of Stokes vector of RCP light scattered in water solutions of polystyrene microspheres with three particle sizes shown on the Poincaré Sphere by crosses, the circles show the results of MC modeling for the same optical properties of scattering samples and geometry of experiment.

To further establish this technique we conducted feasibility experiments using samples of cancerous and healthy kidney tissue from human patients, chosen for its relative uniformity compared with other human tissue types. Samples were biopsied from living patients and set in paraffin wax with one side exposed for observations. Shown here in Figure 5 are the averaged polarization states of detected light scattering from healthy (lower) and cancerous (upper) samples complete with standard deviation.

With a RCP circular incident state of polarization we can see on the Figure 5 that the ellipticity (latitude on the sphere) is higher for the cancerous samples. This is expected to be a result of the increased average size of cell nuclei (as can be 


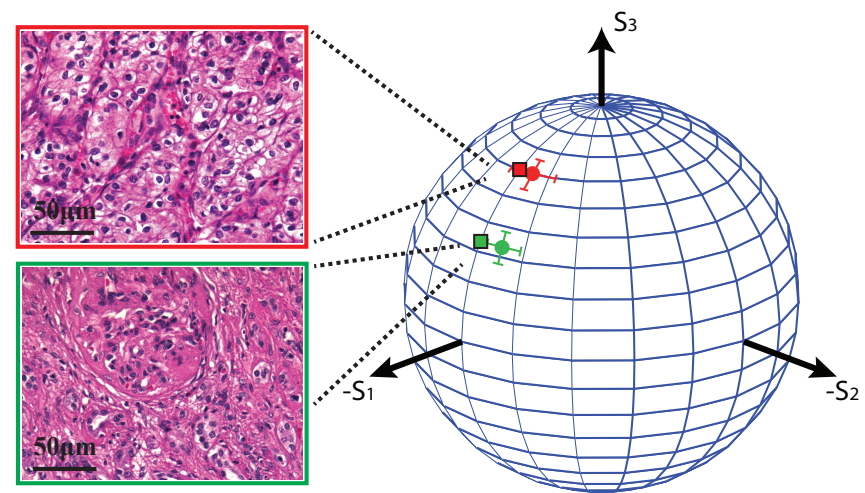

Figure 5. The Stokes vector of light scattered from healthy kidney tissue (lower circle) and cancerous kidney tissue (upper circle) plotted on the Poincaré sphere with corresponding microscope images. Also plotted are the simulated polarizations for healthy kidney tissue (lower square) and cancerous kidney tissue (upper square). Incident light is right handed

seen in the corresponding microscope images, see Figure 5). This leads to higher anisotropy and an increase in successive forward scattering events contributing to the signal where co-polarized helicity dominates. Also included on the figure is the MC simulation results using approximated medium properties for each tissue type and system geometry equivalent to that in Figure 2. The simulation shows very good agreement with the experimental results, and helps demonstrate that alterations to biological tissues are resolvable using the specific geometry of the polarization measurement system.

\section{SUMMARY AND CONCLUSIONS}

Alternative studie ${ }^{20}$ that utilize linear polarized light for probing the structure of living epithelial cells in situ, also demonstrated changes to the signature of scattered light with the increase of cells size in cancerous samples of tissues. The approach presented here can avoid the limitations associated with fast depolarization of linear polarised light due to multiple scattering in such media consisting of large scattering particles. Furthermore a link between anisotropy of scattering and the ellipticity of detected light has been outlined which does not require full Muller matrix characterization. As a result we have a method which has the potential to categorize bulk morphology data of an array of different tissue types in real time. By analogy to the diffusing-wave spectroscopy ${ }^{21}$ we call this approach diffusing-wave polarimetry, and illustrate its utility in diagnostic purpose by probing cancerous and non-cancerous tissue samples in vitro. Diffusing-wave polarimetry provides a good foundation for future work implementing non-invasive diagnostic techniques for early disease detection. The key is understanding within this context the dependence of scattering on the size, shape, refractive index, and number density of scattering particles. Once this is accomplished, categorizing of certain tissue types can begin and the knowledge can be used in practical situations for medical diagnostics.

\section{ACKNOWLEDGMENTS}

The financial support of the Health Research Council of New Zealand (HRC) and the Maurice Wilkins Centre (MWC) is gratefully acknowledged. Authors also acknowledge financial support provided by the University of Otago, New Zealand. AFP thanks Consejo Nacional de Ciencia y Tecnología CONACYT México, for the scholarship provided, in basis of the Program "Sabbatical and Postgraduate Foreign Stay".

\section{REFERENCES}

[1] Ghosh, N. and Vitkin, I.A., "Tissue polarimetry: concepts, challenges, applications and outlook," J. Biomed. Opt. 16, 110801 (2011).

[2] Barron, L.D., [Molecular Light Scattering and Optical Activity]. Cambridge Press, London, UK (1982).

[3] Azzam, R.M.A. and Bashara, N.M., [Ellipsometry and Polarized Light]. Elsevier, Amsterdam, NL (1987).

[4] Bicout, D., Brosseau, C., Martinez, A.S. and Schmitt, J.M., "Depolarization of multiply scattered waves by spherical diffusers: influence of the size parameter," Phys. Rev. E 49, 1767 (1994). 
[5] Hielscher, A.H., Mourant, J.R. and Bigio, I.J., "Influence of particle size and concentration on the diffuse backscattering of polarized light from tissue phantoms and biological cell suspensions," Appl. Opt. 36, 125 (1997).

[6] Rojas-Ochoa, L.F., Lacoste, D., Lenke, R., Schurtenberger, P. and Scheffold, F., "Depolarization of backscattered linearly polarized light," J. Opt. Soc. Am. A 21, 1799 (2004).

[7] MacKintosh, F., Zhu, J., Pine, D. and Weitz, D., "Polarization memory of multiply scattered light," Phys. Rev. B 40, 9342-9345 (1989).

[8] Xu, M. and Alfano, R.R., "Random walk of polarized light in turbid media," Phys. Rev. Lett. 95, 213901 (2005).

[9] Kim, Y.L., Pradhan, P., Kim, M.H. and Backman, V., "Circular polarization memory effect in low-coherence enhanced backscattering of light," Opt. Lett. 31, 2744 (2006).

[10] Kim, A.D. and Moscoso, M., "Backscattering of circularly polarized pulses," Opt. Lett. 27, 1589 (2002).

[11] D. H. Goldstein, [Polarized Light]. CRC Press, Taylor \& Francis Group, Boca Raton (2011).

[12] Ishimaru, A., [Wave Propagation and Scattering Media]. Academic, New York (1978).

[13] Doronin, A. and Meglinski, I., "Online object oriented Monte Carlo computational tool for the needs of biomedical optics," Biomed. Opt. Express 2, 2461-2469 (2011).

[14] Doronin, A. and Meglinski, I., "Peer-to-Peer Monte Carlo simulation of photon migration in topical applications of biomedical optics," J. Biomed. Opt. 17, 090504 (2012).

[15] Kuzmin, V.L. and Meglinski, I.V., "Coherent effects of multiple scattering for scalar and electromagnetic fields: MonteCarlo simulation and Milne-like solutions," Opt. Commun. 273, 307-310 (2007).

[16] Kirillin, M., Meglinski, I., Sergeeva, E., Kuzmin, V.L. and Myllyla, R., "Simulation of optical coherence tomography images by monte carlo modeling based on polarization vector approach," Opt. Express 18, 21714-21724 (2010).

[17] Kuzmin, V.L. and Meglinski, I., "Numerical simulation of coherent back-scattering and temporal intensity correlations in random media (overview)," Quantum Electron. 36, 990-1002 (2006).

[18] Pancharatnam, S., "Generalized theory of polarization and it's applications," Proc. Indian Academy of Sciences A 44, 5 (1956).

[19] Macdonald, C. and Meglinski, I, "Backscattering of circular polarized light from a disperse random medium influenced by optical clearing," Laser Phys. Lett. 8, 324328 (2011).

[20] Gurjar, R., Backman, V., Perelman, L.T., Georgakoudi, I., Badizadegan, K., Itzkan, I., Dasari, R., and Feld, M.S., "Imaging Human Epithelial Properties with Polarized Light Scattering Spectroscopy," Nat. Med. 7, 1245-1248 (2001).

[21] Pine, D.J., Weitz, D.A., Chaikin, P.M. and Herbolzheimer, E., "Diffusing-Wave Spectroscopy,” Phys. Rev. Lett. 60, 1134 (1988). 